\title{
Prevalence Rate of Vulvovaginal Candidiasis and Identification of Candida Species in Women in Referred to Hamedan Hospitals 2013 - 2014, West of Iran
}

\author{
Reza Habibipour ${ }^{1, *}$ \\ ${ }^{1}$ Department of Microbiology, Faculty of Basic Sciences, Hamedan Branch, Islamic Azad University, Hamedan, IR Iran \\ "Corresponding author: Reza Habibipour, Department of Microbiology, Faculty of Basic Sciences, Hamedan Branch, Islamic Azad University, Hamedan, IR Iran. E-mail: \\ habiby.reza@gmail.com
}

Received 2015 July 10; Accepted 2015 November 16.

\begin{abstract}
Background: Vulvovaginal candidiasis is a common gynecological finding among the women worldwide. Objectives: In this study determine of prevalence rate of vulvovaginal candidiasis and identification of Candida species was investigated.

Patients and Methods: In this cross-sectional study study, 350 females selected and examined by wet mount and culture procedure. Results: The prevalence rate, by means of culture procedure was $26 \%$. Candida albicans was responsible for $81.3 \%$ episodes of volvovaginal candidiasis.

Conclusions: In order to decrease the prevalence rate of vulvovaginitis, more epidemiological surveillance and accurate informative programs about public health care, symptoms and signs and transmission ways should be performed in the future.
\end{abstract}

Keywords: Genital Diseases, Vulvovaginitis, Vulvovaginal Candidiasis, Candida albicans

\section{Background}

Candida is ubiquitous fungus known as a normal microflora of skin, gastrointestinal and urinogenital tracts, which could change from asymptomatic to symptomatic. There are many risk factors for development of vulvovaginal candidiasis, like vaginal ecosystem, pregnancy, hormonal contraception, diabetes, stress recent antibiotic use, dietary practices, gastrointestinal colonization by the organism, clothing and weaken immune-compromised system $[1,2]$. Every year millions of women visit gynecologic office, health centers and clinics due to common urinary tract and vaginal problems which most of their complaint refers to vulvovaginal candidiasis [2]. It has been estimated that approximately $75 \%$ of women experience at least one episode of vulvovaginal candidiasis in their lifetime and $40 \%-50 \%$ of them will have recurrences [ 1 , 2]. About $80 \%-90 \%$ of vulvovaginal candidiasis cases are caused by Candida albicans, meantime other species could result in vulva-vaginal symptoms, as well, which medical resistance is the most problematic challenges [3].

Symptoms of vulvovaginal candidiasis are nonspecific and encompass pruritus (itching), soreness; thick and cheesy white vaginal discharge, erythema, edema and dyspareunia $[1,4]$. However, none or just one of these symptoms may observe in vulvovaginal candidiasis. Moreover mixed infections (Bacterial, Trichomonas besides Candida) of most patients lead to misdiagnosis and therapy failures $[4,5]$.

\section{Objectives}

The aim of this study was determination of prevalence rate of vulvovaginal candidiasis amongst Hamedan women referral to hospitals, as it had been reported a significant increase of candidiasis.

\section{Patients and Methods}

The source population of this this cross-sectional study consisted of 350 women referred to Atiyeh and Fatemiyeh, as major obstetrics and gynecology hospitals in Hamedan, for routine examinations, annually cancer and infections tests. The laboratory experiments carried out at the microbiology laboratory of Hamedan branch, Islamic Azad University from October 2013 to February 2014. Patients received an information sheet and signed a consent former grading data protection. A structured questionnaire used to obtain information regarding personal and social status, hygiene behavior, contraceptive method and vulvovaginal candidiasis symptoms (itching, soreness, vaginal 
secretions, inflammation, rashes etc.) of patients. Sampling was simple in an improbable way. In each speculum examination, duplicate High Vaginal Swabs (HVS) were collected using sterile cotton tipped swabs form posterior and lateral cervical of womb, meantime the status of vagina and cervix were recorded. Swabs cultured on Sabouraud Dextrose agar, Sabouraud Dextrose agar with Cyclohexamide and Chloramphenicol. Presumptive Candida species level identification was done by using CHROMagar media based on differences in color, hypha morphology and chlamydospore formation and surface appearance of colonies [6]. The duplicate swab was examined microscopically by $\mathrm{KOH}$ wet mount and Gram staining, for the presence of budding yeast cells and pseudo-hypha of Candida species.

Results were analyzed by statistical tests such as Z-test and Goodness of fit with P values $<0.05$ were considered statistically significant. All of our analysis was done by SPSS-16.

\section{Results}

The result of vulvovaginal candidiasis prevalence among 350 patients, with mean age of 32.2 years, were accordingly in wet mount $21.1 \%(\mathrm{n}=74)$, Gram staining $21.7 \%(n=76)$ and culture procedure $26 \%(n=91)$. The Goodness of fit revealed significant differences associated with different age groups $(\mathrm{P}=0.03)$, as women of childbearing age had the highest rate of vulvovaginal candidiasis. Of the total case documentations obtained about different kinds of contraception methods, $23.4 \%(\mathrm{n}=82)$ were using oral contraceptive pills, 5.7\% $(\mathrm{n}=20)$ injection, $8.9 \%(\mathrm{n}=$ 31) IUD, $13.2 \%(n=46)$ condom, $11.4 \%$ tubectomy, $12.3 \%(n=$ 43) withdrawal method and $23.7 \%(n=83)$ other methods, while $1.4 \%(n=5)$ were non-contraceptive users. Our statistical analysis revealed no significant association of the contraception methods and overall positivity culture rate $(P=0.03)$. However, the highest rate, $28 \%(n=23)$ of positive culture were observed in oral contraceptive pill method (Figure 1). Separately, the trend of infection in women with clinical symptoms more pronounced than those whom have not any symptoms $(\mathrm{P}=0.03)$. The severity of clinical signs and symptoms in women with positive vulvovaginal candidiasis culture were itching, soreness, itching and soreness simultaneously, discharge and itching, and discharge and soreness respectively. Although, there were not observed any significant differences between symptoms (Figure 2). Complementary tests have done on 91 isolates of yeast in order to determine the fungal species. These results indicated C. albicans (81.3\%), the most frequent species, of all cases of vulvovaginal candidiasis in positive cultures, followed by Candida glabrata
(11\%), Candida tropicalis (4.4\%), Candida kuresi (2.2\%) and some other species (1.1\%).

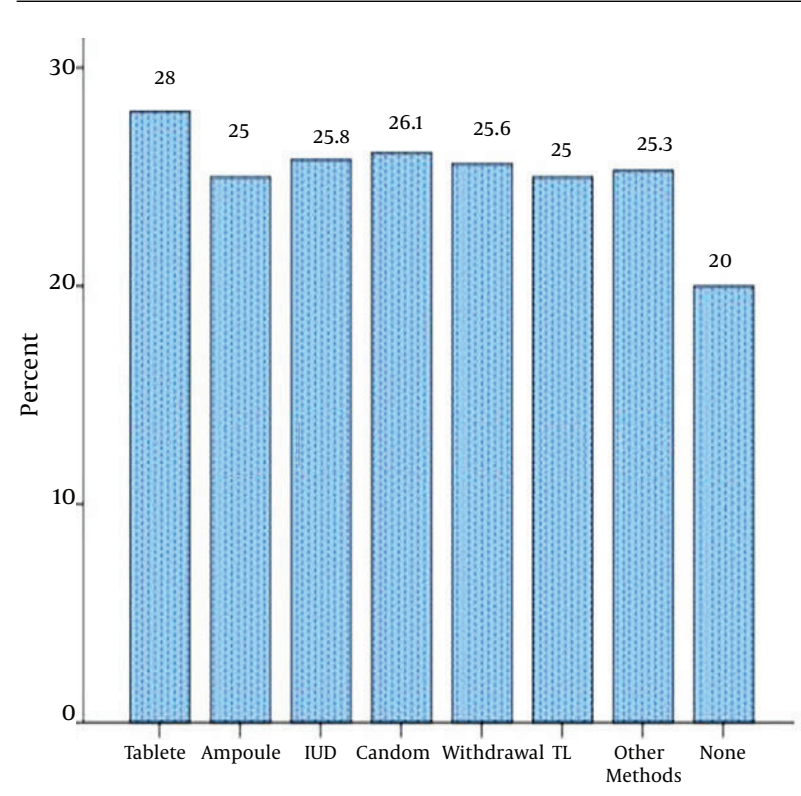

Figure 1. The Frequency Percentage of Vaginal Candidiasis Among the Women Admitted to Woman's Hospitals in Hamedan With Regard to Contraception Methods

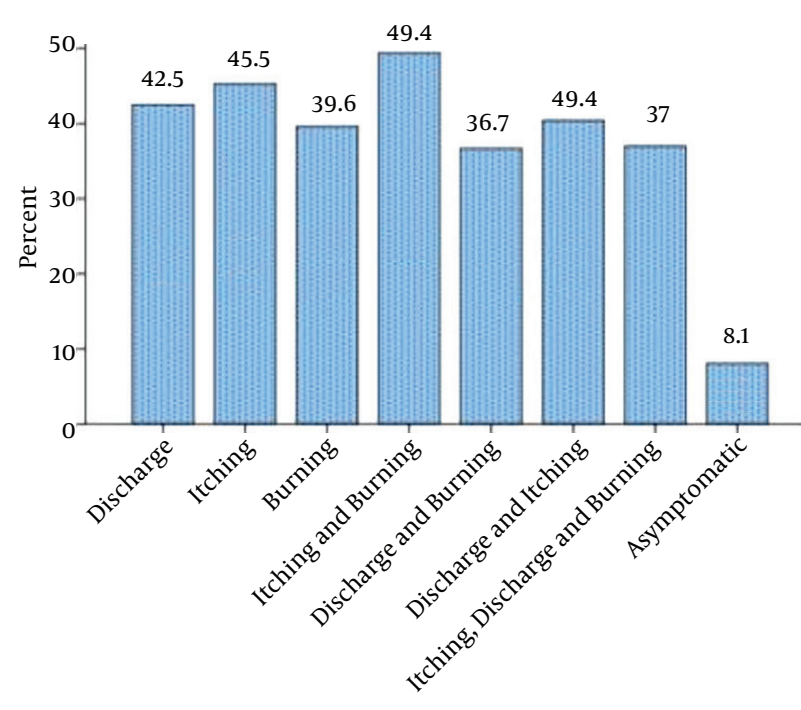

Figure 2. The Frequency Percentage of Vaginal Candidiasis Among the Women Admitted To Woman's Hospital in Hamedan With Regard To Clinical Signs

\section{Discussion}

The results of the present study indicate that the prevalence rate of vulvovaginal candidiasis among 350 of 
women with an mean age of 32.2 years using the wet mount is $21.1 \%$, while it is $21.7 \%$ using the Gram staining method and $26 \%$ using the cultivation method.

The prevalence rate of $26 \%$, observed in cultivation procedure was more than direct procedure, wet mount and Gram staining, which proves more sensitiy of culture. Culture as a gold standard in confirming negative diagnoses, detecting false-negative results of microscopy, arid identifying isolates of Candida species other than C. albicans [2]. High vaginal swabs cultures in the present study, revealed vulvovaginal candidiasis in $26 \%$ of women as compared to $21.7 \%$ on Grams stain and $21.1 \%$, of wet mount ( $\mathrm{KOH}$ preparation). Similarly Levett confirmed higher sensitivity of culture rather than $\mathrm{KOH}$ wet mount and Gram stain [7]. Therefore, a reliable diagnosis can not be made based on Grams stain/KOH mount alone without the collaborative evidence of culture report. Numerous studies performed to establish the frequency of the most common infectious agents for vaginitis, which have shown Candida species rates vary between $2.2 \%-30 \%$ [8]. It could be seen in the present work that prevalence rate of vulvovaginal candidiasis of this study (26\%) compatible with those in other publications. For example in Iran Moallaei et al. studying 213 women with vaginitis, found Candida in 30\% [9]. Meanwhile, findings of Buscemi et al. 28.4\% were near to our finding [10]. In the other hand this finding do not compare well with that of other countries such as Adad et al. 17.3\% - 50\% (during four decades), and Murta et al. 17.8\% which may be related to the climatic, geographical and cultural living conditions $[11,12]$.

In this study, which was similar to other studies like Grigoriou et al. the most separated species from the patients was C. albicans. In the diagnosis of different Candida species, four main species of Candida were isolated [6]. It could be seen in the present work that C. albicans (81.3\%) predominantly and then C.glabrata (11\%), C.tropicalis (4.4\%), C. kuresi $(2.2 \%)$ were identified as the Candida pathogens in culture tests. A similar distribution of Candida spp. was reported by Corsello et al. that C. albicans (77.1\%), C. glabrata (14.6\%), C. kuresi (4\%) and was near to Nazeri et al. findings as well, C. albicans (77.7\%), C. glabrata (19.4\%) and C. krusei (2\%) and other species $(0.7 \%)[3,13]$. This finding does not match well with other reported findings from the point of species and frequencies. Nyirjesy et al. findings was C. albicans (72\%), C. glabrata (11.7\%), C. parapsilosis (8.5\%), C. lusitaniae $(0.8 \%)$ and other species (7\%) [14].

Analysis revealed significant association of the detection rate of vulvovaginal candidiasis with the subject age groups as it was parallel to reported results of Akinbiyi et al. which mentioned age distribution of vulvovaginal candidiasis rate is around childbearing period [5]. It has been found that different kinds of contraception could be a risk factor for vulvovaginal candidiasis infection as others demonstrated it. However, we did not find any significant correlation between the occurrence of vulvovaginal candidiasis and different kinds of contraceptive methods. Although the increased risk for vulvovaginal candidiasis of oral contraceptive (28\%) rather other methods observed, which confirms the observation of Parhizkar et al. [15]. Among the various symptoms, the occurrence of itching was higher which is in agreement with those of other authors [2-4]. The burning sensation that stands as the second major complaint, in this study and some other reports which have been contribute to metabolism of yeast and sometimes due to vulvar skin infection $[2,3]$. In conclusion as microscopy fails to diagnose Candida, and some bacterial and tricomonas symptoms are simillar to candidiasis and since proper diagnosis is the basis for correct treatment, it is recommended to use a culture procedure in cases before therapy. On the other hand, we could not ascertain measurable certainty of medico-social associated factors such as intake of oral contraceptive pills or other forms of family planning methods and vulvovaginal candidiasis rate. The clinical signs and symptoms of vulvovaginal candidiasis caused by $C$. albicans or other Candida species tend to be similar. In our series, we did not show any striking difference between patients with vulvovaginal candidiasis symptoms when compared to existence reports. These findings necessitate more epidemiological surveillance and accurate Informative programs about public health care, symptoms, signs, and transmission ways.

\section{Acknowledgments}

This paper, Coded d/7957, is approved by the research deputy office of Islamic Azad university, Hamedan branch. I acknowledge my special gratitude to Leila Moradi-Haghgo dear colleague in the microbiology laboratory of Islamic Azad University, Hamedan branch.

\section{Footnote}

Funding/Support: This study was supported by Islamic Azad university, Hamedan branch.

\section{References}

1. Jombo GTA, Opajobi SO, Egah DZ, Banwat EB, Akaa PD. Symptomatic vulvovaginal candidiasis and genital colonization by Candida species in Nigeria. J Public Health Epidemiol. 2010;2(6):147-51.

2. Ahmad A, Khan AU. Prevalence of Candida species and potential risk factors for vulvovaginal candidiasis in Aligarh, India. Eur J Obstet Gynecol Reprod Biol. 2009;144(1):68-71. doi: 10.1016/j.ejogrb.2008.12.020. [PubMed: 19261369]. 
3. Corsello S, Spinillo A, Osnengo G, Penna C, Guaschino S, Beltrame A, et al. An epidemiological survey of vulvovaginal candidiasis in Italy. Eur J Obstet Gynecol Reprod Biol. 2003;110(1):66-72. [PubMed: 12932875].

4. Karaer A, Boylu M, Avsar AF. Vaginitis in Turkish women: symptoms, epidemiologic - microbiologic association. Eur J Obstet Gynecol Reprod Biol. 2005;121(2):211-5. doi: 10.1016/j.ejogrb.2004.11.030. [PubMed: 16054964].

5. Akinbiyi AA, Watson R, Feyi-Waboso P. Prevalence of Candida albicans and bacterial vaginosis in asymptomatic pregnant women in South Yorkshire, United Kingdom. Outcome of a prospective study. Arch Gynecol Obstet. 2008;278(5):463-6. doi: 10.1007/s00404-008-0593-8. [PubMed: 18299865].

6. Grigoriou O, Baka S, Makrakis E, Hassiakos D, Kapparos G, Kouskouni E. Prevalence of clinical vaginal candidiasis in a university hospital and possible risk factors. Eur J Obstet Gynecol Reprod Biol. 2006;126(1):121-5. doi: 10.1016/j.ejogrb.2005.09.015. [PubMed: 16256258].

7. Levett PN. Aetiology of vaginal infections in pregnant and nonpregnant women in Barbados. West Indian Med J. 1995;44(3):96-8. [PubMed: 8560888].

8. Mendez M, Calderon J, Soria A, Yui M, Apaza N. Bacterial vaginosis: diagnosis and prevalence in a health center [in Spanish]. Revis Peru Ginecol Obstet. 2015;47(1):58-61.

9. Moallaei H, Mirhendi SH, Brandao J, Mirdashti R, Rosado L. Compari- son of enzymatic method rapid yeast plus system with RFLP-PCR for identification of isolated yeast from vulvovaginal candidiasis. Iranian J Basic Med Sci. 2011;14(5):443-50.

10. Buscemi L, Arechavala A, Negroni R. [Study of acute vulvovaginitis in sexually active adult women, with special reference to candidosis, in patients of the Francisco J. Muniz Infectious Diseases Hospital]. Rev Iberoam Micol. 2004;21(4):177-81. [PubMed:15709796].

11. Adad SJ, de Lima RV, Sawan ZT, Silva ML, de Souza MA, Saldanha JC, et al. Frequency of Trichomonas vaginalis, Candida sp and Gardnerella vaginalis in cervical-vaginal smears in four different decades. Sao Paulo Med J. 2001;119(6):200-5. [PubMed: 11723534].

12. Murta EF, Souza MA, Araujo Junior E, Adad SJ. Incidence of Gardnerella vaginalis, Candida sp and human papilloma virus in cytolog ical smears. Sao Paulo Med J. 2000;118(4):105-8. [PubMed: 10887386].

13. Nazeri M, Mesdaghinia E, Moravej SAR, Atabakhshiyan R, Soleymani F. Prevalence of vulvovaginal candidiasis and frequency of Candida species in women [in Persian]. J Mazandaran Univ Med Sci. 2012;21(86):254-62.

14. Nyirjesy P, Alexander AB, Weitz MV. Vaginal Candida parapsilosis: pathogen or bystander?. Infect Dis Obstet Gynecol. 2005;13(1):37-41. doi: 10.1080/10647440400025603. [PubMed: 16040326].

15. Parhizkar S, Moshfe AA. Prevalence of cervicovaginal infections among the Pap smears of women, Yasuj, 1999 -2000 [in Persian]. Armaghan Danesh. 2003;7(28):37-44. 